\title{
Validation of enzyme-linked immunosorbent assay for measurement of faecal cortisol in fish
}

\author{
Samuel J Lupica \& John W Turner, Jr. \\ Department of Physiology and Pharmacology, University of Toledo College of Medicine,Toledo, OH, USA
}

Correspondence: J W Turner, Department of Physiology and Pharmacology, University of Toledo College of Medicine, Health Science Campus, 3000 Arlington Avenue, Mail Stop 1008,Toledo, OH 43614-2598, USA. E-mail: John.Turner@utoledo.edu

\begin{abstract}
Quantification of glucocorticoid (GC) levels in faeces has become an established method for the non-invasive assessment of adrenocortical activity. These hormones are frequently determined in plasma samples as parameters of adrenal activity and response to stress. Because GCs are metabolized and excreted with both intact hormone and their metabolites present in faeces, the concentration of GCs can be measured in excreta. Faecal samples present the advantages of easy collection, no stress to the animal and elimination of the issue of potentially misleading acute GC spikes. The aim of this study was to determine if an enzyme-linked immunosorbent assay (ELISA) for cortisol was appropriate for monitoring adrenocortical activity in faecal casts of fishes. Performance of the cortisol ELISA was validated by comparison to high-performance liquid chromatography, which is an established method for measuring free GCs and GC metabolites in faeces. Parallelism and sample extraction efficiency were compared for the two methods. Pearson's correlation across samples for these methods was 0.996. Results demonstrated that the ELISA was an efficient, sensitive and reliable method for cortisol measurement in faecal extracts, which should permit integration of non-invasive stress monitoring into studies of fish behaviour and physiology.
\end{abstract}

Keywords: faecal, cortisol, fish, stress, ELISA, HPLC

\section{Introduction}

Endocrine activity in an extensive range of species, including fish, can be significantly altered under stressful circumstances contributed by both natural and anthropogenic factors (Carballo, Jimenez, de la Torre, Roset \& Munoz 2005). Fish subjected to various stressors exhibit primary stress responses similar to mammals, including the release of cortisol from interrenal tissue (equivalent of the mammalian adrenal cortex) (Axelrod \& Reisine 1984). Under chronic stress conditions (e.g., environmental deterioration), longterm mild to moderate cortisol hypersecretion may occur (van den Heuvel \& O'Halloran 2005). Specific physiological consequences of chronic stress include inhibition of innate immunity (Watanuki, Yamaguchi \& Sakai 2002), growth (Jentoft, Aastveit, Torjesen \& Andersen 2005) and reproduction (Berg, Westerlund \& Olsson 2004). Quantification of faecal glucocorticoid (GC) metabolites has become a useful method for the noninvasive assessment of adrenocortical activity in temperamental, dangerous, rare or poorly accessible species (Wasser, Hunt, Brown, Cooper, Crockett, Bechert, Millspaugh, Larson \& Monfort 2000; Turner Jr., Nemeth \& Rogers 2003; Scott \& Ellis 2007). While GCs, and especially cortisol, have frequently been determined in plasma as an indicator of stress, blood sampling is itself invasive and stressful. In addition, faecal samples amortize hormone levels across time, eliminating the issue of potentially misleading acute hormonal spikes associated with sampling plasma (Turner Jr. et al. 2003).

Glucocorticoids are metabolized and excreted with both intact hormones and their metabolites present in faeces (Touma, Sachser, Mostl \& Palme 2003). The main metabolites of GCs are 17- $\beta$-hydroxycorticoids, with a smaller amount (5-10\%) metabolized to 17-ketosteroids, and approximately $10 \%$ is excreted as free hormone (Chelini, Souza, Cortopassi, Felippe 
\& Oliveira 2006). These proportions are relatively stable, making measurement of free hormone a direct and reliable endpoint. Unlike the sulphate- and glucuronide-conjugated forms of faecal glucocorticoids, free glucocorticoids are almost insoluble in water and are readily extractable from faeces in organic solvents. Free cortisol present in faecal samples and maintained at $28{ }^{\circ} \mathrm{C}$ in seawater remains $100 \%$ intact for $>8 \mathrm{~h}$ and $93 \%$ intact after $16 \mathrm{~h}$ (Turner Jr. et al. 2003). The aim of this study was to determine whether an enzyme-linked immunosorbent assay (ELISA) for cortisol could be applied to measurement of faecal cortisol in fish. Cortisol monitoring of fish in both aquaculture and natural settings is a potentially valuable tool. Performance of the cortisol ELISA was validated by comparison to high-performance liquid chromatography (HPLC), which has been used for measuring free GCs and GC metabolites in faeces of numerous mammalian species (Wasser et al. 2000) and more recently in fish (Turner Jr. et al. 2003). Tests for parallelism and extraction efficiency were used to compare the cortisol ELISA and HPLC in faecal casts of parrotfish.

\section{Materials and methods}

\section{Animal model}

The fish faecal samples used in this study were obtained from adult male and female stoplight and queen parrotfish (Sparisoma viride and Scarus vetula respectively) located within reefs of Great Lamesur Bay, Saint John, US Virgin Islands. These samples were part of a separate study involving faecal collections across several years from fish inhabiting this bay (Turner Jr. et al. 2003). Samples were collected via SCUBA and snorkeling at the same time of day to minimize possible influences of diurnal cortisol concentrations. Because sufficient material was not obtainable from a single faecal cast for the HPLC portion of the study (Turner
Jr. et al. 2003), each cortisol value was obtained from a pool of samples from six different individuals.

\section{Extraction}

Desiccated faecal casts for the ELISA (0.2 g) or HPLC $(0.5 \mathrm{~g})$ were mixed in $12 \mathrm{~mL}$ spectrophotometericgrade dichloromethane $\left(\mathrm{MeCl}_{2}\right.$, Curtin Matheson Scientific, Houston, TX, USA) and shaken for $60 \mathrm{~min}$ on a motorized shaker (Burrel, Pittsburg, PA, USA) at $25^{\circ} \mathrm{C}$ and then centrifuged at $1500 \mathrm{~g}$ for $10 \mathrm{~min}$. Ten millilitres of the liquid layer was combined with $1 \mathrm{~mL}$ of $1.0 \mathrm{M}$ sodium hydroxide in a glass scintillation vial, shaken, allowed to separate and frozen on dry ice. Eight millilitres of the $\mathrm{MeCl}_{2}$ layer was withdrawn, aliqouted ( $4 \mathrm{~mL}$ each) into two $12 \times 75 \mathrm{~mm}$ borosilicate glass tubes, evaporated to dryness and stored at $-40{ }^{\circ} \mathrm{C}$ until reconstituted. Dry samples were reconstituted in ethanol and enzyme conjugate (for ELISA) or acetonitrile and distilled water (ACN, for HPLC) for assay (Fig. 1). Ethanol was used in the ELISA due to the denaturing effect of ACN on proteins and its propensity to dissolve the plastic microtitre plates.

\section{Reconstitution for ELISA}

Extracted cortisol samples (from $0.2 \mathrm{~g}$ dessicated faecal material) were mixed with $50 \mu \mathrm{L}$ ethanol (EtOH) and vortexed for $2 \mathrm{~min}$. Fifty-microlitre increments of aqueous enzyme conjugate were added and vortexed for an additional 2 min each to give a final volume of $500 \mu \mathrm{L}$ (see "Cortisol ELISA"). Fifty-microlitres of each sample (in duplicate) was added to the ELISA plate.

\section{Reconstitution for HPLC}

Extracted cortisol samples (from $0.5 \mathrm{~g}$ dessicated faecal material) were mixed with $100 \mu \mathrm{L} \mathrm{ACN}$ and vor-

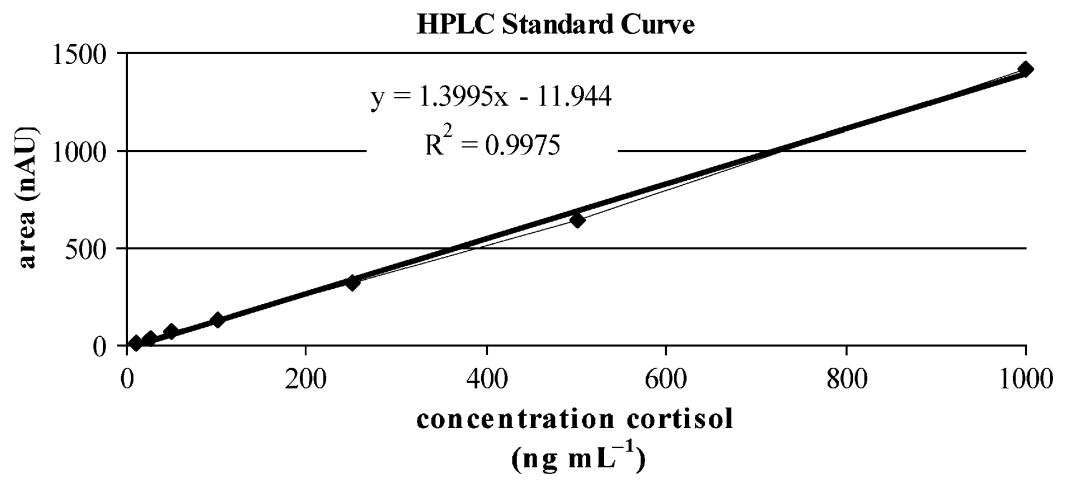

Figure 1 A representative high-performance liquid chromatography (HPLC) cortisol standard curve. The standard curve was derived from seven known concentrations of cortisol (5$\left.1000 \mathrm{ng} \mathrm{mL}^{-1}\right)$ in $10 \%$ acetonitrile $(\mathrm{ACN}) / 90 \% \mathrm{dH}_{2} \mathrm{O}$. 
texed for $2 \mathrm{~min}$. One hundred-microlitre increments of distilled water were added and vortexed for an additional $2 \mathrm{~min}$ each to give a final volume of $1.0 \mathrm{~mL}$. A $250 \mu \mathrm{L}$ aliquot of each sample was loaded onto the chromatography column.

\section{Cortisol ELISA}

A sandwich-type ELISA, modified from methods described by Munro and Lasley (1988), was adapted for determination of fish faecal cortisol. The assay employed a cortisol-horseradish peroxidase (HRP) ligand and antiserum (Antibody R4866; C.J. Munro, University of California at Davis) and commercial cortisol standards (hydrocortisone reference standard; Sigma-Aldrich, St Louis, MO, USA). The polyclonal antiserum was raised in rabbits against cortisol-3-carboxymethyloxime (CMO), linked to bovine serum albumin and cross-reacts with cortisol (100\%), prednisolone (9.9\%), prednisone (6.3\%), cortisone $(5 \%)$ and $<1 \%$ with androstenedione, androsterone, corticosterone, desoxycorticosterone, 11-desoxycortisol, 21-desoxycortisone and testosterone (Munro \& Lasley 1988). The ELISA was completed in 96-well microtitre plates (Nunc-Immuno, Maxisorp Surface; Fisher Scientific, Pittsburgh, PA, USA) layered $24 \mathrm{~h}$ beforehand with cortisol antiserum $\left(50 \mu \mathrm{Lwell}^{-1}\right.$; diluted 1:8500 in bicarbonate coating buffer; $0.05 \mathrm{M}$ $\mathrm{Na}_{2} \mathrm{CO}_{3} / \mathrm{NaHCO}_{3}, \mathrm{pH}$ 9.6). Plates were sealed tightly with waterproof plate sealer covers and incubated overnight at $4{ }^{\circ} \mathrm{C}$. On the day of the assay, the enzyme conjugate (cortisol-3-CMO:HRP) was diluted to 1:20 000 in phosphate buffer (0.1 M PBS containing 0.1\% BSA, pH 7.0). Non-bound antibody was removed from the wells of the microtiter plates with wash solution $(0.15 \mathrm{M} \mathrm{NaCl}$ containing $0.05 \%$ v:v Tween 20 ), blotted and allowed to dry at room temperature. Fifty microlitres of phosphate buffer was pipetted across the entire plate followed by $50 \mu \mathrm{L}$ of cortisol-3-CMO:HRP conjugate solution containing cortisol standards or a reconstitute of extracted faecal sample. Stock cortisol standards stored in ethanol were dried under air and reconstituted in $50 \mu \mathrm{L}$ ethanol and $450 \mu \mathrm{L}$ diluted enzyme conjugate to give a standard range of 5-1000 $\mathrm{mL}^{-1}$. In addition, recovery of cortisol standards added to cortisol-free (previously cortisol extracted) faecal samples was determined. Dried, extracted faecal samples were reconstituted similarly and $50 \mu \mathrm{L}$ of each sample were pipetted in duplicate for the ELISA. Plates were covered tightly with plate sealer covers and the competitive reaction allowed to proceed for $2 \mathrm{~h}$. To separate free from bound hormone, the plates were emptied, rinsed three times with wash solution, blotted and allowed to dry at room temperature. One hundred microlitres of freshly prepared substrate solution (0.05 M citrate, $1.6 \mathrm{mM}$ hydrogen peroxide, $0.4 \mathrm{mM}$ 2,2-azino-di-3-ethylbenzithiazoline sulphonic acid diammonium salt (ABTS, pH 4.0) was then added to all wells and colour was allowed to develop to determine the amount of conjugate (cortisol:HRP) bound to the solid-phase antibody. The colour change was stopped after approximately $60 \mathrm{~min}$ by the addition of $100 \mu \mathrm{L}$ stop solution (0.15 M hydrofluoric acid containing $0.006 \mathrm{M}$ $\mathrm{NaOH}$ and 0.001M EDTA, pH 3.3). Absorbance was measured at $405 \mathrm{~nm}$ with a Bio-Rad Model 550 automatic microtitre plate spectrophotometer (Hercules, CA, USA) and the data were transferred to an interfaced computer for analysis (Gateway model E4300, Irvine, CA, USA). Cortisol concentrations are expressed as nanograms per gram of dry faeces $\left(\right.$ ng g $^{-1}$ ).

\section{HPLC}

Although details of this procedure for faecal cortisol have been previously reported (Turner Jr. et al. 2003), a brief description is provided for the present context. Free cortisol in fish faecal materials was determined by reverse-phase (HPLC, Dionex, Sunny vale, CA, USA) utilizing a standard $3.9 \times 300 \mathrm{~mm} \mathrm{C-18} \mathrm{col-}$ umn (Waters, Milford, MA, USA) and a variable wavelength UV detector (Dionex) set at $240 \mathrm{~nm}$. Before sample analysis, a water blank was run until the column was free of major peaks and a reference standard containing cortisol [hydrocortisone reference standard; Sigma-Aldrich; $50 \mu \mathrm{L}$ in 10\% ACN/90\% water] was run to verify retention times. The flow rate was $1 \mathrm{~mL} \mathrm{~min}^{-1}$ and the elution gradient changed from $10 \% \mathrm{AcN} / 90 \%$ water to $90 \% \mathrm{ACN} / 10 \%$ water over a period of $25 \mathrm{~min}$ (ensuring complete separation of sample compounds) and returned to the initial concentration over another $5 \mathrm{~min}$. Additionally, the run was continued for $15 \mathrm{~min}$ at the initial concentration to re-acclimate the column. The standard curve was developed by HPLC runs of duplicate samples of seven known cortisol concentrations (ranging from 5-1000 $\mathrm{ng} \mathrm{mL}^{-1}$ ).

\section{Results}

\section{HPLC}

Cortisol was readily detectable and measured by the HPLC analysis and the elution time was 

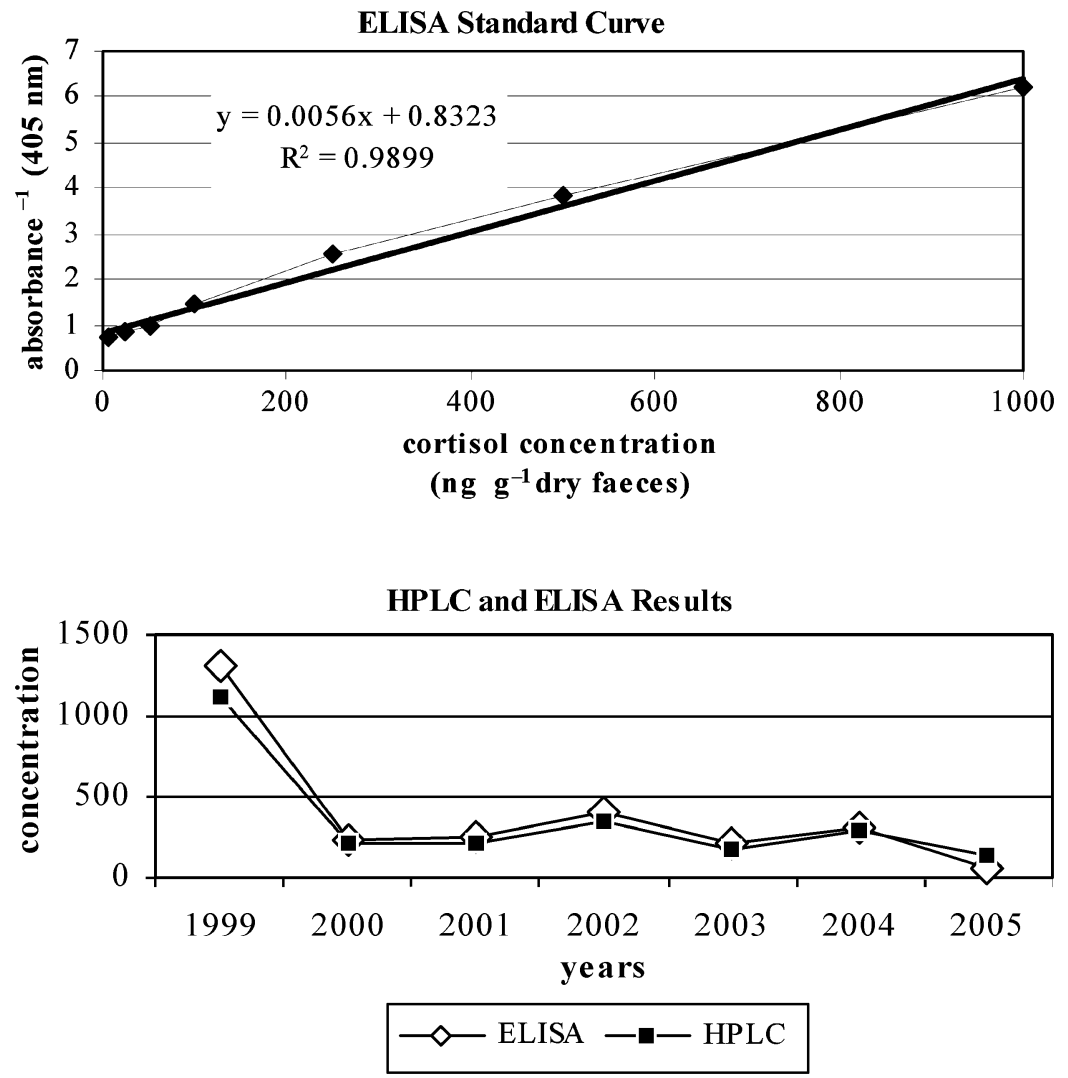

Figure 2 A representative enzyme-linked immunosorbent assay (ELISA) cortisol standard curve. The standard curve was derived from seven known concentrations of cortisol (5-1000 $\mathrm{ng} \mathrm{mL}^{-1}$ ) in enzyme conjugate.

Figure 3 Comparison of high-performance liquid chromatography (HPLC) and enzyme-linked immunosorbent assay (ELISA) results from parrotfish faecal samples from 1999 through 2004. The Pearson correlation coefficient for these data was 0.996 .

$11.91 \pm 0.02 \mathrm{~min}$. The lower limit for cortisol detection in the HPLC portion of this study was $1.8 \mathrm{ng}$ in a $250 \mu \mathrm{L}$ sample loaded onto the chromatographic column. Hormone values are reported as ng cortisol/g dry faeces. HPLC standards ranged from 5 to $1000 \mathrm{pg} \mu \mathrm{L}^{-1}$ in seven incremental amounts (Fig. 1). The HPLC-assayed cortisol values (ng g $^{-1}$ of dry faeces) ranged from 1118.5 for the 1999 sample to 169.5 for the 2003 sample.

\section{ELISA}

A representative ELISA standard curve is shown in Fig. 2. ELISA-derived cortisol values (ng ${ }^{-1}$ dry faeces) were based on the same concentration as for HPLC with $R^{2}=0.9899$ for the calculated curve (Fig. 2). Standard curves for cortisol measured directly and measured after addition to and extraction from cortisol-free faeces were not different. The lower limit using ELISA was $1.0 \mathrm{ng}$ in the usual $50 \mu \mathrm{L}$ sample placed in each microplate. ELISA-assayed cortisol values (ng $\mathrm{g}^{-1}$ of dry faeces) ranged from 1314.2 for the 1999 sample to 210.7 for the 2003 sample.

\section{ELISA-HPLC comparison}

Faecal cortisol assayed by both HPLC and ELISA across the sampling years 1999 to 2004 yielded nearly identical patterns of cortisol levels (Fig. 3). A Pearson correlation coefficient performed on these data was 0.996.

\section{Discussion}

To date there have been no reports of the measurement of cortisol in fish faeces. Radioimmunoassay and HPLC are the most commonly used methods for plasma cortisol measurement in fish Wasser et al. (2000) have reported faecal cortisol measurement by ELISA in terrestrial mammals. The present study has shown that cortisol in fish can be measured in faeces by ELISA and this assay exhibits equal reliability and much greater efficiency and sensitivity than HPLC. On a per-millilitre basis, the ELISA, showed ninefold greater sensitivity than HPLC, which reduces faecal sample weight required for reliable assay $(\mathrm{HPLC}=0.5 \mathrm{~g}$, ELISA $=0.2 \mathrm{~g}$ ). From both per-sample assay time and personnel cost perspectives, the 
ELISA is more practical and less expensive than HPLC analysis. The time required for a single faecal analysis via HPLC is approximately $45 \mathrm{~min}$, whereas an ELISA plate can accommodate up to 96 samples (48 in duplicate) in $<4 \mathrm{~h}$. Finally, the equipment cost for ELISA is $<5 \%$ of that for HPLC. We conclude that the faecalcortisol ELISA validated in this study shows high potential as a sensitive, reliable and cost-effective tool for non-invasive stress assessment in fish.

\section{Acknowledgments}

We wish to thank Dr V. Basrur (Medical University of Ohio) and Dr M. Kim (University of Toledo) for assistance with the HPLC portion of the study and J. Clark for laboratory assistance in the ELISA portion. This study was funded by the Trustus Foundation and the University of Toledo College of Medicine.

\section{References}

Axelrod J. \& ReisineT.D. (1984) Stress hormones: their interaction and regulation. Science 224, 452-459.

Berg A.H., Westerlund L. \& Olsson P.E. (2004) Regulation of Arctic char (Salvelinus alpinus) egg shell proteins and vitellogenin during reproduction and in response to 17beta-estradiol and cortisol. General and Comparative Endocrinology 135, 276-285.

Carballo M., Jimenez J.A., de la Torre A., Roset J. \& Munoz M.J. (2005) A survey of potential stressor-induced physiological changes in carp (Cyprinus carpio) and barbel (Barbus bocagei) along theTajo River. Environmental Toxicology 20, 119-125.

Chelini M.O., Souza N.L., Cortopassi S.R., Felippe E.C. \& Oliveira C.A. (2006) Assessment of the physiologic stress response by quantification of fecal corticosteroids. Journal of the American Association for Laboratory Animal Science 45, 8-11.

Jentoft S., Aastveit A.H., Torjesen P.A. \& Andersen O. (2005) Effects of stress on growth, cortisol and glucose levels in non-domesticated Eurasian perch (Perca fluviatilis) and domesticated rainbow trout (Oncorhynchus mykiss). Comparative Biochemistry and Physiology. Part A, Molecular and Integrative Physiology 141, 353-358.

Munro C.J. \& Lasley B.L. (1988) Non-radiometric methods for immunoassay of steroid hormones. Progress in Clinical and Biological Research 285, 289-329.

Scott A.P. \& Ellis T. (2007) Measurement of fish steroids in water - a review. General and Comparative Endocrinology 153, 392-400.

Touma C., Sachser N., Mostl E. \& Palme R. (2003) Effects of sex and time of day on metabolism and excretion of corticosterone in urine and feces of mice. General and Comparative Endocrinology 130, 267-278.

Turner J.W. Jr., Nemeth R. \& Rogers C.S. (2003) Measurement of fecal glucocorticoids in parrotfishes to assess stress. General and Comparative Endocrinology 133, 341-352.

van den Heuvel M.R. \& O'Halloran K. (2005) Measures of resting immune function and related physiology in juvenile rainbow trout exposed to a pulp mill effluent. Archives of Environmental Contamination and Toxicology $\mathbf{4 8}$, 520-529.

Wasser S.K., Hunt K.E., Brown J.L., Cooper K., Crockett C.M., Bechert U., Millspaugh J.J., Larson S. \& Monfort S.L. (2000) A generalized fecal glucocorticoid assay for use in a diverse array of nondomestic mammalian and avian species. General and Comparative Endocrinology 120, 260275.

Watanuki H., Yamaguchi T. \& Sakai M. (2002) Suppression in function of phagocytic cells in common carp Cyprinus carpio $L$. injected with estradiol, progesterone or 11-ketotestosterone. Comparative Biochemistry and Physiology. Part C, Pharmacology,Toxicology and Endocrinology 132, 407-413. 